\title{
Debates en el campo de la Niñez
}

\section{El Sistema que debería $\operatorname{ser}^{1}$}

\section{Luisina Finos}

Licenciada en Trabajo Social (UNR)

Comisión de Niñez, Colegio de Trabajo

Social, Segunda Circunscripción, Rosario

Correo: lu.finos@hotmail.com
María Sol Santa Cruz

Licenciada en Trabajo Social (UNR)

Comisión de Niñez, Colegio de Trabajo

Social. Segunda Circunscripción, Rosario

Correo: sols19@hotmail.com

\section{Natalia Juárez}

Licenciada en Trabajo Social (UNR)

Comisión de Niñez, Colegio de Trabajo

Social, Segunda Circunscripción, Rosario

Correo: juareznataliaanalia@gmail.com

1 El presente artículo fue presentado en carácter de Ponencia en la XI Reunión de Antropología del MERCOSUR, realizado en Uruguay en el mes de diciembre 2015. 
Resumen

El campo actual de la Niñez, se constituye en un entramado complejo de interrelaciones, que conllevan a relaciones caóticas, conflictivas, complejas, donde el anudamiento no siempre logra disiparse, sino que se constituye como lo característico de las intervenciones en este campo. Desde el análisis de una situación de vulneración de derechos por la que ha atravesado un niño de la Ciudad de Rosario (Provincia de Santa Fe, Argentina), se busca problematizar acerca del Sistema de Protección Integral, que se crea desde la sanción de la Ley 12.967 "Promoción y Protección integral de los Derechos de los Niños, Niñas y Adolescentes", sancionada en el año 2009, analizando principalmente desde la noción de institucionalidad y las lógicas de intervención que se constituyen como instituidas.

\section{Palabras claves}

Niñez - Vulneración - Derechos Institucionalidad - Intervención
Abstract

The current field of childhood constitutes a complex network of interrelationships, which chaotic, conflictive relationships. From the analysis of a situation of infringement of rights by which has passed through a child of Rosario, we put in question the Integral Protection System, created since the enactment of Law 12.967, sanctioned in 2009, mainly analyzing from the notion of institutionality and the logics of intervention that are both constituted and instituted.

Keywords

Childhood - Vulnerability - Rights

- Institucionalism - Intervention 


\section{Introducción}

Luego de la ratificación argentina a la Convención Internacional de los Derechos del Niño (CIDN) y en concordancia con sus principios, se sanciona en nuestro país en el año 2005 la Ley $N^{\circ} 26.061$ de "Protección Integral de los Derechos de Niños, Niñas y Adolescentes", dando lugar a un nuevo paradigma de la niñez.

En la provincia de Santa Fe, cuatro años más tarde se sanciona la Ley Provincial 12.967 de "Promoción y Protección Integral de los Derechos de las Niñas, Niños y Adolescentes"; se sanciona en 2009, su decreto reglamentario - nro. 619 - data de 2010, y la Ley tiene una modificatoria (por Ley 13.237) en 2011.

Nos parece importante realizar una caracterización del denominado Sistema Provincial de Protección Integral de los Derechos de la Niños, Niñas y Adolescentes, en la Provincia de Santa Fe Argentina, para lo que nos serviremos de dicha herramienta jurídica. Es en esta legislación donde se enuncia la conformación del mencionado Sistema, agregando que se trata tanto de organismos gubernamentales como de entidades privadas, para lo que otorga un ordenamiento por "niveles": local, regional y provincial.

En el nivel local, sitúa órganos descentralizados denominados Servicios Locales, siendo éstos unidades técnico-operativas que intervienen en situaciones de amenaza o violación de los derechos de los Niños, Niñas y Adolescentes (en adelante: NNyA), planteando una articulación territorial para el abordaje de estas situaciones.

En el nivel regional, se constituye en un actor una de cuyas funciones es intervenir en coordinación con los servicios locales, en el nivel provincial, actuando la Subsecretaria de Derechos de la Niñez, Adolescencia y Familia y la Direcciones Provinciales de Promoción de los Derechos de la Niñez, Adolescencia (organismo descentralizado de la Subsecretaria) y Familia. La Subsecretaría tiene como funciones: la coordinación del Sistema de Protección Integral, la elaboración de políticas públicas integrales, la elaboración de un Plan Provincial de Promociones y Protección de Derechos, la realización der investigaciones en este campo, la gestión y transferencia de fondos, la inci- 
dencia en la sociedad civil para el reconocimiento de los derechos de los NNyA ${ }^{1}$. La Dirección Provincial de Promoción de los Derechos de la Niñez, Adolescencia, tiene entre sus funciones la coordinación directa de las delegaciones regionales (como ya fue enunciado, el nivel regional) y brindar la asistencia técnica jurídica.

Antes de avanzar en esta caracterización, es importante visualizar algunas consideraciones que se establecen entre los niveles descritos (local /regional/provincial). En principio, para simplificar lo dicho, dentro de estos tres niveles vamos a encontrar la posibilidad de intervención según diferentes grados de responsabilidad, que se enuncian como competencias específicas, donde se simplificaría el Sistema Provincial de Protección Integral de los Derechos de la Niños, Niñas y Adolescentes, en la Provincia de Santa Fe, quedando ordenado sólo en dos niveles de intervención.

Un Primer Nivel de Intervención (lo que sería el nivel local), cuya competencia reside en la aplicación de Medidas de Protección Integral, y un Segundo Nivel de Intervención (lo que sería el nivel regional y provincial), cuya competencia fundamental es la aplicación de Medidas de Protección Excepcional² (no eximiéndose de la posibilidad de aplicación de Medidas de Protección Integral).

Ahora bien, las Medidas de Protección Integral (en adelante, MPI) consisten en preservar o restituir derechos amenazados o vulnerados de los NNyA, cualquiera sea el factor que produzca o incida en esta situación (ya sea que provenga del Estado, la sociedad, los progenitores, el propio NNyA); supone la aplicación de programas, dispositivos y servicios para efectivizar el pleno goce de los derechos ${ }^{3}$. Las

1 Es dable destacar que existen más funciones específicas, que se detallan en la Ley 12.967 (Santa Fe/Argentina), en su Art. 30, resaltándose a los fines de esta presentación sólo algunas funciones de relevancia.

2 Según datos estadísticos del Censo Nacional 2010 (INDEC), en la Provincia de Santa Fe viven 905.029 NNyA, de los cuales 647, se encuentran sin cuidados parentales. En entrecruzamiento de datos con el Relevamiento Nacional - UNICEF 2012, donde se retoman los datos del Censo Nacional del año 2010, y se señala que de esos 647 N,NyA sin cuidados parentales, 140 NNyA se encuentran alojados en Instituciones públicas y 393 en Instituciones privadas, 95 N, NyA se encuentran en cuidado familiar público y 19 en cuidado familiar privado. Estos alojamientos provienen el 93\% por determinación del Órgano Administrativo, y el $7 \%$ por determinación Judicial, cuyos principales motivos se fundan en Abuso Sexual Infantil y Maltrato Familiar.

3 La Ley 12.967 de la Provincia de Santa Fe/Argentina enuncia el Derecho a la Vida (Art. 9), Derecho a la Integridad Personal (Art. 10), Derecho a la Identidad (Art. 11), Derecho a la convivencia familiar y 
Medidas de Protección Excepciona (en adelante, MPE), por su parte, se refieren a la aplicación de aquellas medidas cuando las MPI resulten insuficientes o inadecuadas, implicado la separación de un NN/A de su medio familiar o su centro de vida; poseen una temporalidad acotada (con plazo máximo de un año y seis meses)4, existiendo un control de legalidad (cumplimiento del proceso) y de razonabilidad (proporcionalidad entre los medios y fines buscados) que realiza el/ la Juez/a competente en materia de Familia. La separación del NN/A de su centro de vida, conlleva el alojamiento en: Ámbitos alternativos familiares, ya sea por parentesco, otros miembros de familia ampliada o de la comunidad, formas convivenciales alternativas a su grupo familiar (Instituciones de alojamiento o Familias solidarias) y, por último, Centros terapéuticos de salud mental, en razón de abordaje clínico ante determinadas situaciones.

Es así que se entreteje una red de corresponsabilidades, ya sea del Estado, entidades públicas o privadas, organizaciones no gubernamentales, la sociedad civil, donde se establece la responsabilidad de poner en conocimiento y de abordar la situación de $\mathrm{NNyA}$, cuyos derechos se encuentren amenazados o vulnerados.

A fin de otorgarle mayor consistencia al Sistema, la legislación inaugura la creación de nuevos actores, que cobran gran protagonismo y se constituyen en engranajes fundamentales, en el abordaje de situaciones, en la implementación de políticas públicas y en generar procesos de participación de los NNyA. Se crea el Consejo Provincial de Niñez de carácter consultivo y de asesoramiento, conformado por diversos representantes 5 ; la Defensoría Provincial de Niñas, Niños

comunitaria (Art. 12). Derecho a la salud (Art. 13), Derecho a la Educación (Art. 14, 15 y 16), Derecho a la libertad (Art. 17), Derecho al Descanso, Recreación, Deporte y Juego (Art. 18). Derecho a la Propia Imagen (Art. 19), Derecho a la Libre Asociación, Reunión y Transito (Art. 20), Derecho a Opinar y ser Oído (Art. 21), Derecho al Trabajo de los Adolescentes (Art. 22) y Derecho a la protección contra la Explotación Económica (Art. 23), Derecho a la Seguridad Social (Art. 24).

4 Respecto al plazo establecido en la aplicación de Medidas de Protección Excepcional, luego de la sanción del Código Civil y Comercial Argentino (agosto 2015), se estableció en dicho Código un plano más reducido, refiriendo que el plazo de la separación de un NNyA de su centro de vida no puede exceder los 180 días como plazo máximo a aplicar (Art. 607 - Inc. C). Debiendo, a nuestro entender, adecuarse la legislación provincial a los plazos que establece el Código.

5 El Consejo Provincial de Niñez es presidido por el Subsecretario de los derechos de la Niñez, Adolescencia y Familia, participan representantes de los diversos Ministerios, representantes del Poder Judicial, representantes de Municipios y Comunas, representantes de Organizaciones no 
y Adolescentes bajo la figura del Defensor Provincial de los NNyA, cuya función es asumir la defensa de los derechos de los NNyA y ser auditoria de la aplicación del Sistema de Protección Integral; también se crea una Comisión Interministerial de la Niñez y Adolescencia6, algunas de cuyas funciones principales son garantizar la concreción de acciones de los diversos Ministerios y garantizar la transferencia de recursos.

A modo esquemático, resulta interesante visualizar el Sistema de Protección Integral que la legislación plantea a fin de analizar cómo ha resultado su implementación. Es desde estas caracterizaciones que sostenemos la existencia de un entramado de relaciones caóticas, conflictivas, complejas, donde el anudamiento no siempre logra disiparse, sino que se constituye como lo característico de las intervenciones en este campo. A continuación, desde el análisis de una situación de vulneración de derechos por la que ha atravesado un Niño de la Ciudad de Rosario, se busca problematizar el Sistema de Protección Integral.

\section{Presentación de situación}

El grupo familiar al que haremos referencia se encuentra compuesto por María y sus tres hijos, Juan de 12 años, Luis de 4 años y Paula de 2 años, teniendo éstos diferentes progenitores ${ }^{7}$.

En el año 2013 se realizan las primeras intervenciones del Servicio de Atención Ciudadana (SAC), cuya función se orienta a abordar situaciones familiares o individuales que necesiten una eventual ayuda económica, perteneciente a la Secretaría de Promoción Social de la Municipalidad de Rosario (Organismo Público).

\footnotetext{
Gubernamentales, Universidades y Colegios Profesionales.

6 La comisión Interministerial está conformada por un Subsecretario del Ministerio de Salud, Ministerio de Desarrollo Social, Ministerio de Reforma y Gobierno de Estado, Ministerio de Educación, Ministerio de Trabajo y Seguridad Social, Ministerio de Justicia y Derechos Humanos, Ministerio de Seguridad, Ministerio de Innovación y Cultura, Ministerio de Producción y por ultimo Ministerio de Economía.

7 En la situación presentada, se ha preservado la identidad de los involucrados, por lo que se han alterado los nombres.
} 
En el primer relato, María transmitió que su hijo mayor estaba siendo maltratado física y verbalmente por el padrastro de ella. También relató que fue echada por el dueño de la casa donde se encontraba viviendo, debido a los reiterados maltratos que propiciaba su padrastro a su hijo, situación que generaba la intervención constante de sus vecinos. A partir de esto, tanto la mamá de María como su grupo familiar conviviente se mudan de barrio.

En aquel momento, María manifestó no querer ir a vivir con ellos, argumentando la situación de maltrato a la cual se encontraban expuestos. Desde el SAC, la respuesta que se construyó fue el otorgamiento de alimentos y la gestión por una noche de un alojamiento transitorio; se define luego derivarla junto a sus hijos al centro REMAR (Institución Cristiana, que existe a nivel internacional, cuyo objetivo es la ayuda humanitaria, intentando la salida de personas de situaciones precarias, bajo una lógica de comunidad conviviente) ${ }^{8}$ con el compromiso de realizar búsqueda de alguna vivienda, como así también volver a referenciarse con las instituciones del territorio, fundamentalmente el centro de salud y la escuela, ya que le permitiría acceder a la Asignación Universal Por Hijo (AUH)9 como también realizar controles necesarios de su embarazo de ocho meses. Cuando se produjo el nacimiento de su tercera hija, continuó siendo alojada en REMAR.

En el marco del objetivo de egreso del grupo familiar del hogar, se evalúo la importancia de acompañar el proceso de búsqueda de la vivienda. Desde REMAR se definió trasladar al grupo familiar a otro hogar, de la misma asociación, ubicado en otro distrito de la ciudad, lo cual implicó derivar la situación a otra profesional del SAC (Equipos organizados distritalmente) ya que la anterior profesional interviniente no tomaba dicha zona. El traslado se planteó de forma urgente, ya que la convivencia se definía como "insostenible”. María

8 Para más información sobre REMAR, visitar su sitio web oficial: http://remarargentina.org/ acerca-de-remar/

9 La AUH, se constituye desde el año 2009, en un Derecho para los NNyA, cuyos responsables adultos no se desempeñen en la economía formal o se encuentren desempleados, percibiendo un monto de dinero mensual, debiendo presentar dos veces anuales controles de salud de los NNyA y certificado escolar. Para más información, ver Ley 24.714. 
seguía sosteniendo que estaba angustiada y que no se adaptaba al hogar. Nuevamente se volvió a reubicar al grupo familiar y se firmó un acta acuerdo, desde el SAC, donde María tendría veinte días para encontrar una vivienda para vivir con sus hijos.

Se comenzó a rastrear la familia ampliada y una vez localizada el grupo familiar se fue a vivir a un nuevo barrio de la ciudad. Es dable destacar que la familia ampliada que se menciona sería el mismo grupo conviviente anteriormente (su progenitora), con el cual se encontraban expuestos a situaciones de maltrato.

Ante este nuevo contexto, comenzó la intervención de la Dirección de Atención de Situaciones de Vulnerabilidadad Social (en adelante, DASVS) perteneciente también a Promoción Social Municipal, quien realizaría el seguimiento de la situación debido a que ya contaban con domicilio fijo y por la situación de vulnerabilidad en la se encontraba el grupo familiar. En cuanto a esta última Dirección, su función es intervenir ante situaciones de niños y niñas en grave riesgo físico y psíquico por violencia, abusos, situación de calle, consumo de sustancias, explotación sexual, carencia de cuidados parentales, compuesto por Equipos interdisciplinarios, divididos distritalmente.

Entre las acciones realizadas por el equipo de la DASVS, en el marco de la construcción de las medidas de protección integral que establece la legislación vigente, se pueden mencionar las siguientes: programación y acompañamiento de turnos con el efector de salud, problematización acerca de la convivencia con el agresor de su hijo mayor. Se le planteó la posibilidad de gestionar la compra de una vivienda; se realizó acompañamiento en la búsqueda de la misma; se acompañó la tramitación del DNI de sus hijos. Se realizó entrevista con el hijo mayor de María, el cual refirió que salía a cirujear con el padre de su hermano. Se problematizó con María acerca de dicha actividad y se reforzó la importancia de que el niño se incluyera en la escuela. Se realizó incorporación de los niños más pequeños al Centro de Acción Familiar ${ }^{10}$. Se solicitó la incorporación de un acom-

10 Los Centro de Acción Familiar son instituciones territoriales donde se desarrollan diversas actividades inclusivas, destinado principalmente a NN de 3 a 7 años. 
pañante personalizado orientado a reforzar el rol materno de María en relación a los cuidados de sus hijos (ésto no logró sostenerse debido a que el perfil del Acompañante Personalizado no respondía a los requerimientos que la situación ameritaba). Se solicitó al Centro de Convivencia Barrial (CCB) la incorporación de niño mayor al espacio recreativo de ludoteca (el niño asistió esporádicamente). Se acompañó al niño mayor a la escuela del barrio para que iniciara un proceso de aceleramiento para poder incluirlo en el año lectivo 2015. En un primer momento esta acción fracasó; se retomó la propuesta a partir de que colaboradores de un comedor del barrio observaron que el niño buscaba comida en los volquetes de basura y eso permitió acercarse al niño y habilitar no sólo la propuesta inicial sino la concurrencia al comedor.

En el mes de noviembre de 2014, por medio de una comunicación telefónica desde el comedor, se transmitió al equipo de la DASVS que Juan se presentó con un golpe en el ojo debido a una pelea con el padre de Luis. Ante este episodio, María realizó la denuncia en la comisaría cercana a su domicilio. Al día siguiente, profesionales del CAF y de la DASVS acompañaron a María junto a Juan para que él fuese revisado por el médico forense.

Se definió, junto a los equipos intervinientes territorialmente (CAF, CCB y el centro de salud, DASVS), solicitar adopción de una medida de protección excepcional. El objeto de aplicación de dicha medida se fundó en la necesidad de resguardar a los niños de situaciones cotidianas de vulneración de derechos donde los adultos no lograban garantizar cuidados básicos necesarios para el desarrollo, el derecho a la salud, la educación, la alimentación, la estimulación y el hecho de no poder tomar los diferentes espacios ofrecidos para trabajar la situación.

La medida excepcional se efectivizó en 2015, el lugar de alojamiento para Juan fue una institución perteneciente al Sistema de Protección Integral que cuenta con convenio con el Organismo de aplicación de la Ley 12.967, el Hogar de Protección del Menor (HO. PRO.ME); Luis y Paula fueron alojados por el Programa de Familia 
Solidaria, esto es, familias que alojan transitoriamente a Niños y Niñas.

El equipo de la DASVS sostuvo las visitas a Juan una vez por semana garantizándole la vestimenta y las actividades que realizaba por fuera del lugar de alojamiento. Para estas actividades, el niño contó con un acompañante personalizado, el cual fue gestionado posteriormente a la toma de la medida. El direccionamiento de las estrategias de éste fue realizado por el equipo de la DASVS. Con el programa de Familia Solidaria se realizaron comunicaciones telefónicas a los fines de organizar los encuentros entre los hermanos, los cuales se sostenían en HOPROME de dos a tres veces por semana.

En relación a los acuerdos inter-institucionales en el abordaje de la situación realizados por el equipo de la Dirección Provincial de Promoción de los Derechos de la Niñez, Adolescencia y Familia, se puede hacer referencia de los siguientes acuerdos: en el mes de febrero de 2015, entrevistas con los niños, comunicación con el centro de salud, entrevista con María. Desde el Programa de Familia Solidaria se acuerda visitar a la familia que alojaba a Luis y Paula y trabajar la posibilidad de que Juan pudiese ir a visitar a sus hermanos. Desde la DASVS se acuerda solicitar información a la escuela, visitar a Juan y el armado de actividades para él junto con su acompañante.

En el mes de marzo de 2015, se vuelve a realizar un acta interinstitucional en donde se acuerda que desde el equipo social del hogar donde se encontraba alojado Juan, se gestionara el pago de transporte para garantizarle la asistencia a la escuela. Desde el DPPDNAYF volverían a solicitar un acompañante para Juan, ya que quien estaba había renunciado y averiguarían sobre una posible evaluación de salud mental para María. En cuanto al Programa de Familia Solidaria, iniciarían un espacio de juegos, cada 15 días, en el ámbito de la DPPDNAYF. En el mes de abril de 2015, Juan se retiró de forma voluntaria de la institución de alojamiento, regresando a convivir con su madre y la pareja de ésta.

Luego en reunión mantenida con todos los actores intervinientes, se trasmitió desde el Equipo de la DPPDNAyF, que los Niños que se encontraban conviviendo en Familia Solidaria, también regresarían a 
convivir con su familia de origen, siendo esto dispuesto sin acuerdo de todas las instituciones intervinientes por el Organismo facultado para la adopción de la Medida de Protección Excepcional.

\section{Análisis de la situación planteada}

En principio, nos parece interesante recorrer la situación analíticamente apelando a algunas categorías para visualizar que, si bien existe un acuerdo en el sistema de protección integral de la Provincia de Santa Fe, acerca de concepciones como la integralidad y la concepción de vulneración de derechos, al retomarse en las acciones concretas éstas no recobran sentido, sino que se vacían de contenido y se traducen en estrategias que no conllevan a una recuperación de los derechos vulnerados por los que atravesaron los $\mathrm{NNyA}$, sino que se generan nuevas vulneraciones.

Un primer concepto del que vamos a servirnos es el de dispositivo, siguiendo a Agambem (2011): "Lo que trato de indicar con este nombre es, en primer lugar, un conjunto resueltamente heterogéneo que incluye discursos, instituciones, instalaciones arquitectónicas, decisiones reglamentarias, leyes, medidas administrativas, enunciados científicos, proposiciones filosóficas, morales, filantrópicas, brevemente, lo dicho y también lo no-dicho, éstos son los elementos del dispositivo. El dispositivo mismo es la red que se establece entre estos elementos (...) por dispositivo, entiendo una especie — digamos- de formación que tuvo por función mayor responder a una emergencia en un determinado momento. El dispositivo tiene pues una función estratégica dominante (...) El dispositivo está siempre inscripto en un juego de poder (...) Lo que llamo dispositivo un caso mucho más general que la episteme. O, más bien, la episteme es un dispositivo especialmente discursivo, a diferencia del dispositivo que es discursivo y no discursivo". Es desde esta mediación conceptual que podemos leer la complejidad de la situación, en tanto develamiento de aquellas líneas de fuerza que se van trazando, como juegos de poder, donde 
se inscribe lo institucional, la territorialidad, la construcción de la demanda y los procesos de intervención que se van implementando.

Ahora bien, el sistema de protección integral de Niñez, se encuentra conformado por un conjunto de instituciones que no logran conformar una red articulada, es así como al analizar la situación podemos observar la acción de diversos actores, el Servicio de Atención Ciudadana (cuyo enfoque es la ayuda económica), el Equipo de la Dirección de Atención de Situaciones de Vulnerabilidad Socia (cuyas acciones están orientadas a lograr el acceso de derechos de los NNyA como ser la educación y la salud), el Equipo de la Dirección Provincial de Promoción de los Derechos de la Niñez, Adolescencia y Familia (cuya intervención sólo se habilita en el marco de la aplicación de una Medida de Protección Excepcional), el Centro de Convivencia Barrial y Centro de Acción Familiar (orientados a incorporar a los Niños y Niñas a determinadas actividades recreativas) y REMAR (cuya acción es brindar alojamiento desde una fuerte lógica de recuperación), Partido Político (siendo su enfoque la adquisición de una vivienda para el grupo familiar), donde la fragmentación de las intervenciones es lo que constituye, es lo que va configurando lo que se enuncia como una Medida de Protección Integral.

Visualizamos un conjunto de acciones que se van construyendo al interior de cada equipo de trabajo, donde se va produciendo una objetivación del Otro, donde se configura e instala una universalización de la infancia, y donde lo que se busca es la normatividad de la familia (con las consecuencias que eso acarrea cuando el Otro es puesto en falta en todo momento), sin intentar - muchas veces por la urgencia- enriquecer la mirada desde lecturas del contexto con atravesamientos históricos, culturales, políticos y económicos.

Entendemos la Medida de Protección Integral como aquella construcción que posibilita a un Otro, en tanto sujeto de derecho, una real efectividad de un derecho (superando su declamación enunciativa). Ahora bien, para que esta Medida habilite a un Otro, debe estar atravesada por construcciones que no sólo se focalicen en los derechos de los Niños, Niñas y Adolescentes, sino también en toda la conformación familiar, por lo que entendemos que una MPI sólo 
puede ser reparadora en tanto fortalezca y restituya derechos (y no sólo derechos, sino trayectorias de vida y procesos emancipatorios), tanto de los adultos como de los NNyA.

Otra de las características que se evidencian, es lo que podríamos denominar "enquistamiento institucional" (que forma parte sin duda de la fragmentación de la MPI), donde las acciones planteadas para llegar a tales fines deben efectivizarse por el hecho de la propuesta misma. En la situación planteada podemos citar como ejemplos el planteamiento de la posibilidad de gestionar la compra de una vivienda; otra acción ejemplificadora podría ser la inscripción de uno de los niños a una institución barrial, donde territorialmente se ofrece, se gestiona, se acompaña, pero no recobra el sentido planteado y ésto no logra instalarse. Es ahí donde cabe interrogarse acerca de las instituciones, generadas hacia adentro, con escaso margen para pensarse desde el movimiento, confundiendo muchas veces la construcción de institucionalidades con su conformación edilicia. Desde esta concepción de institucionalidades, se trasmite (implícita y explícitamente) una narrativa de los Otros (ajeno a un Nosotros), que están por fuera de las instituciones, que acceden a ellas a través de propuestas pre establecidas, sin margen de elección; "la política debe estar orientada hacia la formación reflexiva de los sujetos de manera que puedan desarrollar su capacidad de decir que no de manera argumentativa y racional. Para ello, la intervención debe proveerles de códigos de información para interpretar alternativas ofrecidas y para escoger con criterios validos" (Matus, 2014:165). Esta posibilidad de decir que no (sin que sea leído desde la falta o desde una perspectiva sancionatoria), es uno de los ejes que dota de sentido procesos de ciudadanización, no estando presente esta posibilidad en los abordajes territoriales del Sistema de Protección Integral de Niñez.

Otro concepto interesante a retomar es el de integralidad ya que aporta a una visión superadora y un giro político frente a los modelos segmentados del abordaje técnico-metodológico presentes en las políticas sociales, específicamente las políticas de niñez, y en las intervenciones en lo social, poniendo el acento en la interdependencia de los derechos y en la horizontalidad del poder del conocimiento. 
La integralidad, conceptualmente, está vinculada a los modos de ver y nombrar las manifestaciones de la cuestión social y cómo se define la satisfacción de un derecho y los efectos que tiene sobre el ejercicio de otros derechos. Lo cual obliga a superar la fragmentación de las prestaciones sociales, económicas y culturales.

Coincidimos con Baratta (1999) en que uno de los principales logros de la perspectiva de derechos ha sido el de contribuir a clarificar que el desarrollo de la niñez no es un asunto de la órbita privada mientras que lo público sería la protección de ésta. Si partimos de esta definición es imprescindible restablecer la primacía de las políticas sociales básicas como plataforma del Sistema de Protección Integral de los Derechos de la Infancia. Esto significa que las políticas públicas universales deben constituirse en el "gran integrador" y que, con respecto a éstas, las políticas específicas deben instituirse en complementarias y restitutivas. Entendemos por complementarias a las intervenciones que propician la promoción de los derechos específicos de niñas y niños, la problematización de las necesidades, la socialización de las prácticas de cuidado hacia los NNyA y el acceso a prestaciones de servicio especiales. Por restitutivas, aludimos a las acciones que protegen los derechos de niñas y niños de las amenazas y violaciones por parte de terceros, del Estado o del propio niño o niña (Art. 33 a 41 Ley 26061). Las mismas se encuentran fortalecidas y orientadas a través de una serie de normas específicas que dan forma a un grupo particular de derechos a la protección proactiva o reactiva por parte del Estado a esas violaciones. En el andamiaje institucional creado por las leyes de promoción y protección de derechos, el organismo de aplicación debe consensuar un plan de acción que permita implementar en forma transversal con las diferentes áreas del Estado los objetivos propuestos. A su vez, se contempla entre sus variadas funciones que pueda planificar y brindar servicios especializados a las niñas, niños y adolescentes y sus familias y diseñar normas generales y principios rectores que deben cumplir las instituciones públicas y privadas.

La articulación de las instancias institucionales requiere de acuerdos de trabajo (marcos precisos y consensuados) que se plas- 
men en guías claras de procedimientos para facilitar la resolución de las situaciones, evitando que los $\mathrm{NNyA}$ sean sometidos a prácticas re-victimizantes.

A su vez, debido a la complejidad de estas actuaciones, es imprescindible problematizar algunas categorías que merecen una discusión interdisciplinaria. La amenaza o vulneración de derechos como categorías que pretenden significar, clasificar, delimitar objetos de intervención requieren de un análisis profundo, ya que en la enunciación universalizante pueden justificarse y repetirse acciones que una orientación garantista de los derechos ha querido desterrar. Tanto se trate de prevenir, revertir o proteger, nos referimos a acciones y acontecimientos evaluados negativamente y nos remite necesariamente a las significaciones y representaciones de los riesgos.

Mitjavila (2006) refiere que el riesgo es un término polisémico de diferentes usos, que se ha convertido en un instrumento abierto a la construcción de múltiples significados sociales. Los discursos sobre el riesgo responden invariablemente al juego de diferentes racionalidades, intereses y pautas culturales que organizan la percepción y las respuestas sociales a esos mismos peligros (Douglas y Widavski, 1992). Se puede reflexionar en diferentes claves de racionalidad en relación a este término tan maleable. Podemos pensar en poblaciones de riesgo y construir los factores que determinan su probabilidad, por lo tanto, las intervenciones van a estar dirigidas a alterar esos factores para modificar o evitar los acontecimientos indeseables.

Desde otras perspectivas, según la antropóloga Mary Douglas (1990), los riesgos son construcciones culturales que se basan en ideales morales y de justicia, en los que se enfatizan algunos aspectos de los peligros y se tienden a ignoran u ocultar otros. Así, puede usarse para legitimar la política o desacreditarla, para proteger a individuos contra las instituciones predatorias o proteger las instituciones contra individuos rapaces. De hecho, el riesgo provee términos seculares para reinscribir las sagradas escrituras.

Al tener en cuenta estas consideraciones, pensamos que la formación de los profesionales que integran los equipos de trabajo para llevar adelante las políticas de infancia, no puede dejar de problema- 
tizar estas nociones ya que en buena parte de ello depende la codificación de la grilla de inteligibilidad de los problemas con estatuto público de niños, niñas y familias.

Ahora bien, luego de analizar algunos ejes que atraviesan la construcción de la Medida de Protección Integral, creemos necesario ahondar en la Medida de Protección Excepcional (MPE) ${ }^{11}$, cuya principal dificultad es su enfoque estrictamente administrativista, es decir que el eje se centra en el cumplimiento del procedimiento administrativo, en detrimento de un enfoque que garantice alojamientos (con todo el significado que conlleva, alojamientos de singularidades, alojamiento de diversidades, alojamiento de complejidades) del NnyA. En tal situación, Rivas (2014) plantea que "priorizar la dimensión del procedimiento implica convertir a la intervención en un mecanismo más de control social"; y es en esta perspectiva que la MPE se vivencia por quienes la atraviesan ( $\mathrm{NNyA}$, grupos familiares, actores con lazo con el $\mathrm{NNyA}$ ) desde una posición sancionatoria, que conlleva a experiencias traumáticas y complejas, difícilmente reversibles.

Otro eje que envuelve a la MPE, es la continuidad de la ampliación de las facultades del Poder Judicial, a través del control de legalidad y razonabilidad, que refiere a la "ampliación de una visión judicializada a la política social y la atribución casi angélica a los jueces y defensores" (Bustelo, 2007). Esto debería ser profundamente revisado, ya que ha configurado todo un dispositivo (como 1 Dirección Provincial de Promoción de los Derechos de la Niñez, Adolescencia y Familia) desde una óptica estrictamente de derechos y esto conduce a incurrir en un nuevo error: creer que la óptica de derechos se erige únicamente desde una perspectiva jurídica, negando lo que debería ser su campo especifico propositivo y efectivo, el de las políticas públicas en materia de Infancia, Adolescencia y Familia.

Es necesario mencionar que en la situación planteada los niños/ as han sufrido una doble separación, en principio de los adultos y

11 La diferenciación en cuanto al análisis categorial que se realiza, respecto de las Medidas de Protección Integral como de las Medidas de Protección Excepcional, en tanto poder identificar algunos ejes prevalecientes, que no implica que atraviesan de modo exclusivo a una como a otra medida, sino que el objetivo es resaltar lo característico. 
también de su grupo de hermanos (dos de ellos alojados en Familia Solidaria y el mayor en una institución de tránsito), donde se evidencia que no ha podido construirse desde el Estado algo del orden del fortalecimiento de los lazos entre los hermanos, donde se descuida, en esta decisión de dar continuidad a viejas lógicas institucionales (que responden a una institucionalización de la infancia), el tipo de cuidado que se construye "una nueva forma de significar el cuidado e interactuar con su construcción social, un factor de afectivación” (Franco y Merphy, 2001:15).

Por último, lo que determina la solicitud de la MPE y su aplicación, tiene su fundamento en la amenaza o vulneración de derechos (irreversible tal situación por medio de la MPI). Por ello, la lectura que se realiza es la de un quiebre intempestivo ante determinada situación, quiebre que indica convocar a más actores para una intervención. Se hace necesario posicionarnos acerca de que entendemos por vulnerabilidad. Según Puebla (2013:1), el "concepto de vulnerabilidad psico-social, refiere a un proceso de condicionamiento social por insatisfacción de una serie de necesidades básicas (vulneración de derechos, sobre todo en las etapas de socialización temprana) que hacen proclives a niños y jóvenes a ser dañados, a quedar fuera del acceso a derechos (educación, salud, contención familiar-comunitaria) a ser socializados en el 'escenario callejero' (deambulación, abandono de sus grupos de crianza, estrategias de supervivencia, explotación laboral, abusos físicos y sexuales) y a enfrentar dificultades con la Justicia y con la Policía en etapas estructurantes de su personalidad. En unos casos son afectados por la desatención desde las Políticas Sociales, por inadecuadas e inoportunas y son 'tutelados' por una justicia 'minoril' que estigmatiza y discrimina, en otros son criminalizados selectivamente a través de un control punitivo autoritario y discriminatorio, en ambos casos se vulneran derechos".

Adscribimos a la mirada de que las vulnerabilidades por las que atraviesan los NNyA, se inscriben en trayectorias de vida, en la construcción de procesos identitarios, en territorialidades, que devienen de procesos históricos, sociales, económicos, políticos y culturales, $\mathrm{y}$ es desde esa lectura en que debe inscribirse el abordaje necesario 
de determinadas situaciones, para que constituyan acciones ancladas en revertir situaciones de vulnerabilidad, si no, son meros apaciguamientos que no revisten modificaciones de fondo.

\section{Aportes finales desde el Trabajo Social}

Desde nuestra profesión debemos comprender la concepción que se tiene de niñez e infancia, dado que "cada sociedad o al menos cada espacio cultural crea una determinada forma de niñez, en el tiempo y espacio" (Ochoa, 1983). Las formas que adquiere la infancia dentro de la sociedad, las ideologías y su construcción social, nos permiten hablar de la infancia como categoría social, y del niño como sujeto de desarrollo y actor social que puede ser definido a partir de su posición dentro de un sistema social y de su participación en el sistema.

Nuestro trabajo de promoción de la infancia en diferentes niveles, basa su intervención en el Enfoque de Derechos que exhorta y faculta a los poseedores de derechos a exigirlos, esto significa que no son vistos ni considerados como objetos, sino como individuos y sujetos sociales que exigen sus derechos legales, por lo cual los niños como personas, como sujetos integrales, requieren de su reivindicación social y para su puesta en práctica debemos de comprometer al Estado y Sociedad Civil.

El Trabajo Social debe trascender las experiencias narradas para la incorporación de nuevos conocimientos, lo cual nos permitirá enriquecer la comprensión de cómo un Estado y una sociedad conciben a la niñez e infancia, pudiendo desde este lugar contribuir a visibilizarla como un actor de la sociedad garantizando la participación y el ejercicio de los derechos de niños, niñas y adolescentes.

Esto nos indica que el rol del Trabajo Social, en el ámbito local, provincial y nacional, en la promoción de la infancia es trascendental e importante, porque permite realizar la gestión del proceso de manera articulada, desde la propuesta e implementación de espacios y mecanismos de atención y protección de la infancia, hasta la promoción a través de políticas sociales desarrolladas a partir de la misma 
realidad y con la participación de los actores sociales, desde el Estado y la sociedad civil.

La defensa y promoción de los Derechos del Niño constituye una reivindicación histórica y social, debido al carácter y situación de la infancia de invisibilidad y de consideración de objeto social; es la redefinición de la cultura de infancia, de la visibilidad social del niño como sujeto de derechos, como sujeto social, de la redefinición de patrones socioculturales y políticos para la atención y promoción de la infancia, desde la sociedad civil para el Estado, desde la revisión y adecuación de normas y leyes existentes, de funciones y roles de las instituciones y organismos, hasta la implementación y desarrollo pleno del Sistema de Protección Integral de Niñas, Niños y Adolescentes.

Recuperamos, por ello, a Haraway cuando plantea que "las luchas sobre lo que será considerado como versiones racionales del mundo son luchas sobre cómo ver". Las luchas de ver y de nombrar son lo que nos permite ir reconociendo nuevas institucionalidades, donde se prioriza el carácter de igualdad en tanto la capacidad de todas las personas (adultos, jóvenes y niños/as) en constituirse en actores sociales y políticos desplegando procesos de conocimiento y de actuación política. Es decir, procesos de ciudadanización donde la mirada se amplía e incorpora a un proyecto societario, donde el problema no es organizativo, sino conceptual y político, como plantea Boaventura de Souza Santos, "está en la capacidad de formular problemas nuevos para los cuales no existe aún la solución” (2000:36).

En tanto profesionales del Trabajo Social, nos encontramos interpelados por esta perspectiva de los derechos humanos constitutiva del campo de la niñez. Se hace necesario y urgente repensar nuestro quehacer profesional, el rol político que jugamos y la intencionalidad de la intervención profesional que históricamente nos fue asignada, es decir, pensarnos a pesar de la lógica dominante, y la profesión cómo participa, genera las trasformaciones sociales acordes a la nueva institucionalidad que la niñez/juventud exige hoy, se trata de una discusión ideológica acerca de por qué estamos ahí, en ese campo especifico. 
Para finalizar, nos serviremos de un intento de conceptualizar la Infancia, entendiéndola como un campo relacional, donde existen relaciones de fuerza, que dan movimiento a dicho campo, lo que lo constituye como dinámico, se trata de una situación por el que atraviesa un sujeto político, con entrecruzamientos políticos, económicos, sociales y culturales donde, siguiendo a Colangelo (2003), refiere valerse de tres categorías necesarias para analizar la infancia: la diversidad cultural, la desigualdad social y la cuestión de género; por lo que esos deben ser los ejes constructores de las Infancias. No se transita esta situación en un contexto de empobrecimiento, no se transita de igual modo en Occidente que en Oriente, no se transita de igual modo siendo Niño que siendo Niña, por lo que trabajar desde un enfoque de derechos implica necesariamente complejizar las miradas y reconocer la diversidad.

\section{Referencias bibliográficas}

AGAMBEN, G. “Qué es un dispositivo?”, en: Revista Sociológica, año 26, № 73, Ciudad de México, Universidad Autónoma Metropolitana-Azcapotzalco, mayo-agosto 2011. Pp. 249-264.

AGAMBEN, G. Infancia e Historia. Buenos Aires, Adriana Hidalgo editora, 2010.

ARIAS, A; GODOY, B. y MANES, R. (Comp.) Debates en torno a la construcción de institucionalidad. Aportes para la reconstrucción de lo público. Buenos Aires, Espacio, 2014.

BUSTELO, E. El recreo de la infancia. Argumentos para otro comienzo. Buenos Aires, Siglo XXI editores, 2007.

BARATTA, A. "Infancia y democracia”, en: Derecho a tener derecho. Infancia, derecho y politicas sociales en América Latina, tomo 4, UNICEF, Montevideo, 1999. Pp. 207-236.

COLANGELO, Ma. A., "La mirada antropológica sobre la infancia. Reflexiones y perspectivas de abordaje" Ponencia presentada en Congreso Internacional: La formación docente entre el S. XIX y S. XXI. Ministerio de Educación, Buenos Aires, 2003.

DE JONG, E; BASSO, R. y PAIRA, M. (comps.). La familia en los albores del nuevo milenio. Buenos Aires, Espacio, 2001.

DE SOUSA SANTOS, B. A crítica da razão indolente. Contra o desperdicio da experiência. Porto Alegre, Afrontamiento, 2000. 
DOUGLAS, M. "El riesgo como recurso forense", en: DAEDALUS. Journal of the American Academy or arts and sciences, 1990.

DOUGLAS, M. y WIDAVSKY, A. Risk and Culture. Oxford, Brasil Blakwell, 1992.

FRANCO, T. y MERHY, E. "El reconocimiento de la producción subjetiva del cuidado", en: Revista Salud Colectiva, vol. 7 Nº 1. Lanús, ene/abr 2001.

MATUS. T. "Trabajo Social y la reconstrucción de lo público”, en: Arias A., Godoy B., Manes, R. (comps.) Debates en torno a la construcción de institucionalidad. Aportes para la reconstrucción de lo público. Espacio, 2014.

MITJAVILA, M. "El riesgo como instrumento de individualización social”, en: BARKÚN, M. y KRMPOTIC, C. El conflicto social y político: grados de libertad y sumisión en el escenario local y global. Buenos Aires, Prometeo, 2006. Pp. 91-108.

OCHOA, J. “La Infancia como Construcción Cultural”. Documentos de Trabajo, $\mathrm{N}^{\circ} 15$, Santiago de Chile, 1983.

PUEBLA, M. D. Democracia y Justicia Penal Juvenil. Doctrina e Intervención. San Juan, EFU/ Universidad Nacional de San Juan, 2005.

RIVAS, S. "Infancia: entre la protección y la administración. Reflexiones en torno a las concepciones de la política de infancia desde la perspectiva de derecho", en: Revista Margen $\mathrm{N}^{\circ} 73$, junio 2014. [En línea: septiembre 2015] Disponible en: www.margen.org/ suscri/margen73/silvinarivas.pdf

ROIGE, M. Niñez, marginalidad y politicas públicas. Análisis de un dispositivo estatal. Buenos Aires, Libros de la Araucaria, 2010.

ROLLET, C. "Del interés del Estado en los Derechos de los Niños", en: Le Groupe Familial, $N^{\circ} 138$. 1993. Pp. 4-11.

\section{NORMATIVAS CONSULTADAS}

Convención Internacional de los Derechos del Niño - 1989.

Ley Nacional $26061-2005$.

Ley Provincial 12967 - 2009.

INDEC - Censo Nacional de Población y Vivienda, 2010.

UNICEF - Relevamiento Argentino, 2012.

Recepción: 22/02/2016

Aceptación: 29/11/2016 